\title{
Feature processing as kernel for integrated CAE systems
}

\author{
F.-L. Krause, E. Rieger, A. Ulbrich \\ Institute for Machine Tools and Manufacturing Technology (IWF) \\ Department of Industrial Information Technology \\ Technical University Berlin \\ Pascalstrasse 8-9 D-10587 Berlin-Germany
}

For the integration of the tasks to be mastered within the product development process it is necessary to take account of non-geometrical information, from design to manufacturing process, alongside geometrical design shape. The introduction of objects as carriers of semantic information leads to the use of features. In the present contribution, a concept and its realization are described that facilitate a flexible definition and computer-internal representation as well as the interpretation of features as semantically endowed objects. Based on the feature model, basic functionalitites for feature processing are introduced, which serve as a common kernel for integrated CAE-Systems.

\subsection{INTRODUCTION}

Increasingly high demands placed on the product development process with respect to the shortening of development and job throughput times, the lowering of development costs and an improvement in product quality have raised the requirement for unbroken continuity in the information-technological linkage of the associated tasks into process chains. Beyond the geometrical description of the component shape, the exchange of semantic information for use in all phases of product development must be supported. To meet these requirements features can be used as semantically endowed objects that accompany the product development process from customer request through to product release. The essential requirement here is the ability to define and process the features in a task- and product-specific fashion and to adapt them flexibly to the changing constraints of 
product development. Parametrical models generated with features support the automation of associated design, configuration and planning processes [BJM 92].

In the present chapter the implementation of feature processing environment is described, which serves as a common kernel for integrated CAESystems to be used in the product development process, Figure 1.

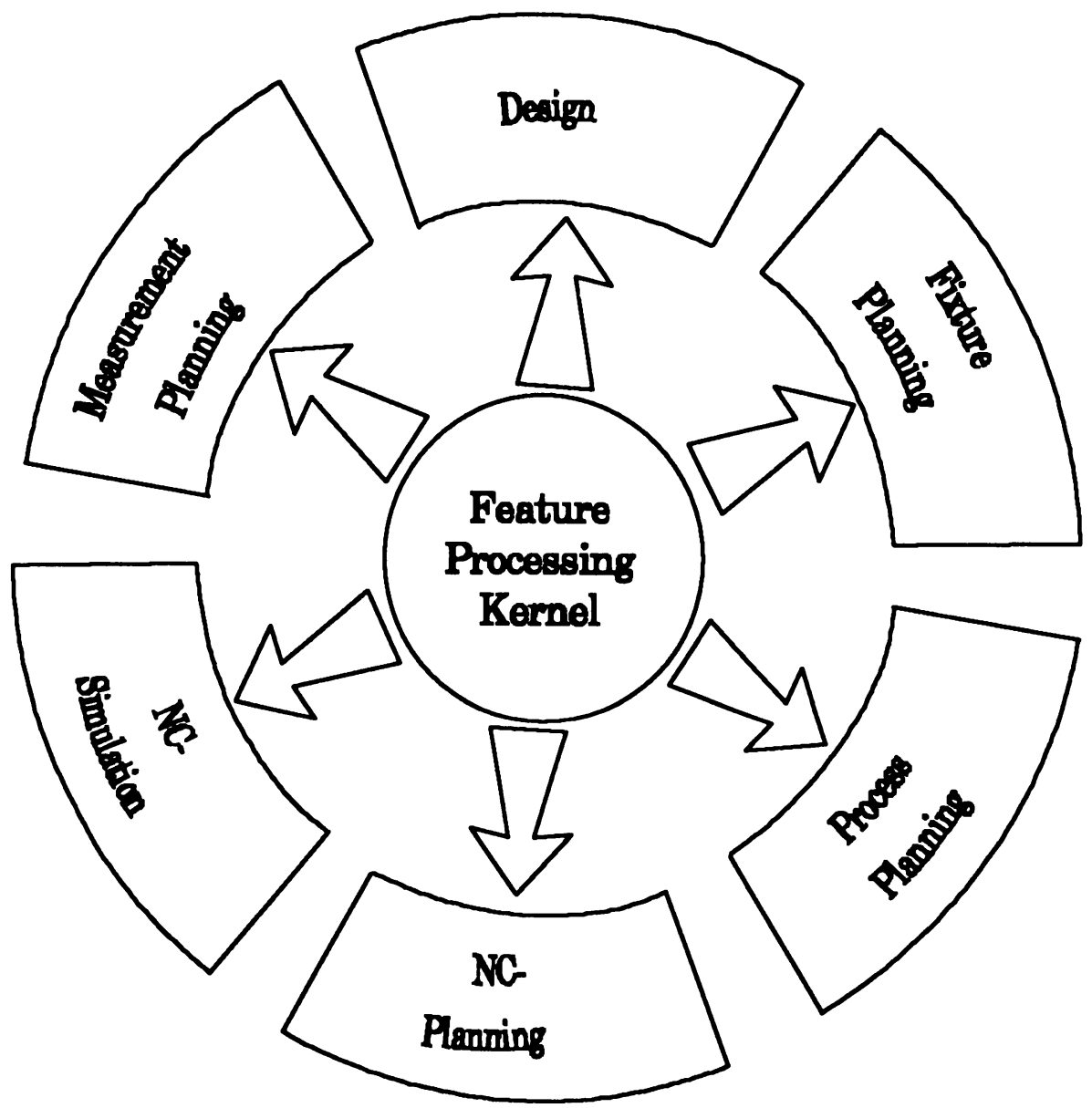

Figure 1. Application Components of the Feature-Based Process Chain

\subsection{FEATURES AS CORE ELEMENTS OF INTEGRATED CAE SYSTEMS}

As the carrier of information within the product development process, features can be employed as semantically endowed objects that accompany product development from the customer request through to product release [SHA 91], [COF 92], [HOU 93]. Correspondingly, the definition of the feature concept 
underlying the feature approach must make possible the integration of semantics into the content described in the feature. A feature is therefore to be defined as [KUV 90]:

Feature := Form Feature \& Semantics

The components of this definition can be explained as follows:

Features, or more precisely semantic features, can be described as geometryoriented objects that are based on three classes of attributes. Static information is designated as a data attribute. Rules and methods determine the behavior of the features. With the aid of relations the interrelations among semantic Features are represented.

Form features are defined as structure-oriented groupings of geometric elements that describe surface formations and sub-volumes without any semantics. They are designated as explicit form features. In contrast to these implicit form features are described procedurally. Each form feature is explicitly represented, but does not always have an explicit representation at its disposal. An essential property of form features is that they can be assigned different semantic features [SRS 90], [REV 89], [SHA 91].

Semantics can be classified based on different application areas within computer-integrated product development:

1 Design-oriented semantics,

2 Manufacturing-oriented semantics, or

3 Quality-oriented semantics.

Corresponding to the orientation of the semantics different types of features result, which will be explained in the following, Figure 2.

\subsubsection{Design Features}

The semantics of design features can be sub-divided into the fields:

1 geometry-oriented semantics, which contain e.g. a classification of the geometrical elements and their relations,

2 technology-oriented semantics, to which parameters for configuration computations are available,

3 functional-oriented semantics, which contain the functional interrelations within an assembly or between its functional surfaces.

Features are regarded as design objects during the product design process. These design objects make a partial modelling possible for the designer that supports his design-related thought processes. The design process proceeds by means of elements that constitute functional units in design terms and that contain the necessary information in as complete a form as possible. 


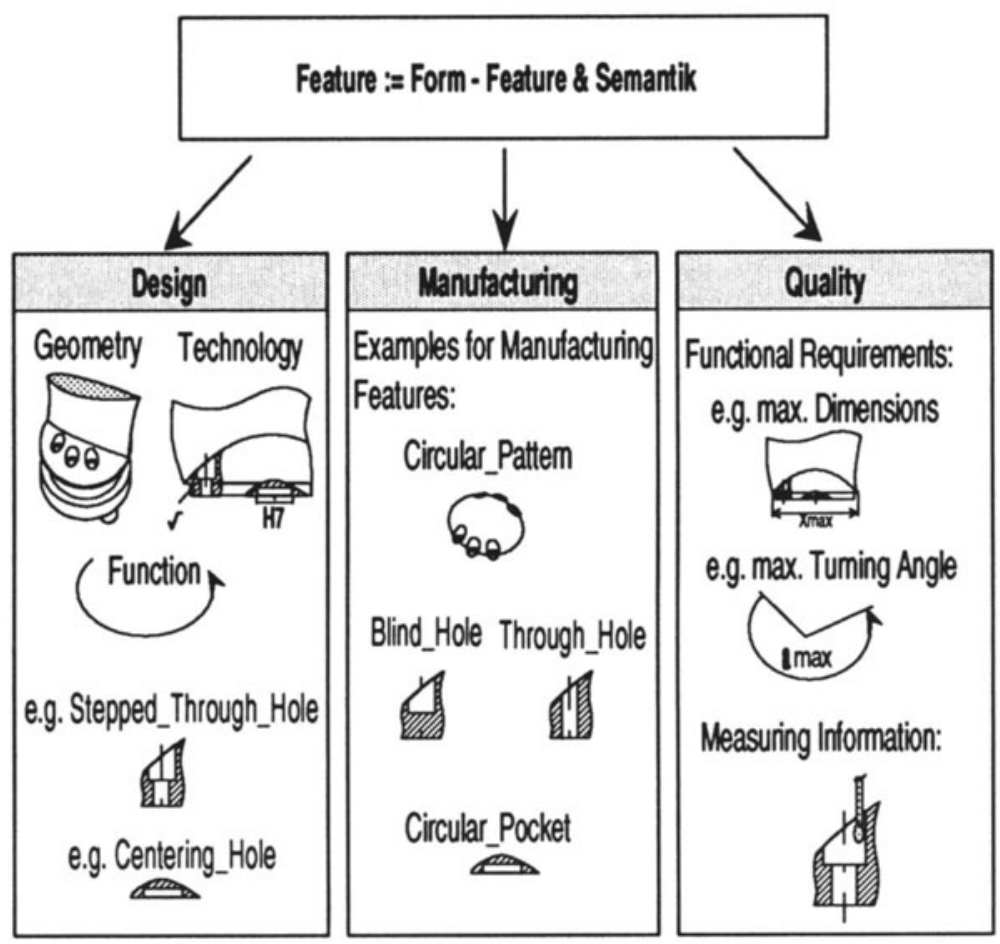

Figure 2. Application Oriented Figure Types

In contrast to conventional CAD systems in which the designer creates his designs using basic geometrical forms without semantics, the elements are now pre-defined in their design-related aspects. Features are to be understood as semantically endowed design objects that, in addition to their technically descriptive data also contain information about their behavior in the design. It is possible for the user to design his own features without being limited in respect of the complexity of the feature. Company-specific features are to be created as required and managed system-independently in a central feature library .

\subsubsection{Manufacturing Features}

The manufacturing-oriented semantics are to be distinguished on the basis of the manufacturing process, e.g. a distinction must be made between sheet metal machining and metal cutting. This expresses the fact that the interpretation of identical form features leads to different results [ALT 91].

A further aspect is the differentiation among features that are processed through the removal of material or that constitute the basis for the configuration of clamping devices. The former are volume-oriented, relate to the differential geometry between blank and finished part, and are derived from the description of the finished part. The latter are surface-oriented and relate at any given time to the current work-piece state after machining. These features cannot be derived from 
the design features or the geometry of the finished part.

\subsubsection{Quality-Oriented Semantics}

Quality features are described in a fashion analogous to the definition cited above by means of a quality characteristic related to a design area. A shape area is represented either by the entire product, a specific assembly, an individual part or a form feature [KUW 93].

Within the framework of preventive quality assurance errors or product requirements are designated as quality-oriented semantics. Errors are e.g. impermissible instances of characteristics of a shape area.

The same definition applies as well for measurement features, which are used for the support of NC planning of coordinate measuring machines. A connection is here established between form features and the quality characteristic tolerance [KRC 94].

\subsection{KERNEL COMPONENTS FOR FEATURE PROCESSING}

For feature modelling, methods are required that support feature processing in an application-independent manner. The task of the feature kernel is the computerinternal representation of the model generated within the given application including the operations employed for generation as well as the provision of semantically endowed product information connected with the representation subject to application-specific requirements. The requirements resulting from the central task of the feature kernel are:

1 flexible application-oriented definitions of features,

2 sufficient power of task-related feature information contents,

3 availability of functionality for the generation and manipulation of the feature model,

4 application-independent representation of features, and

5 representation of information contents and their product-related modelling history [LMM 90].

In order to achieve the greatest possible flexibility with respect to the application-related specification and, at the same time, system independence, the textual language PDGL (Part Design Graphic Language) was developed for the description of generic features [KKR 91]. The use of EXPRESS as syntactic basis provides sufficient linguistic power for the description of information required for product and process modelling [ISO 92a]. Features exist after their graphicinteractive definition independently of the system in their textual form and are interpreted at run-time by the PDGL interpreter contained in the feature kernel. The result of the interpretation is carried over into the corresponding computerinternal representation of the feature kernel. 
In contrast to EXPRESS, which was conceived for the structured description of information within the framework of ISO 10303 (Product Data Representation and Exchange) [ISO 92a] for the standardization of product data exchange, PDGL serves to describe features as product elements and for the definition of functions required for feature instantiation. The PDGL description of a feature thus consists of two blocks. The first block contains the definition of the feature type with its data attributes and sub-features. During the interpretation of this block the feature type is created as an object in the computer-internal representation. This represents the non-geometric part of the feature information and contains references to the associated feature geometry stored in the geometric modeller. The second part is the description of the function that leads to the generation of the specific feature object including its geometric description.

For generation, combination and management of the feature geometry is done by the geometric modeller. In the current implementation the geometric modelling kernel ACIS [BRA 88] is used for these tasks. To preserve the independence of the feature modeller from the geometrical modeller a functional interface was implemented that makes it possible to define and use functions of other application modules as part of the PDGL stock of functions at runtime. To this end a standardized interface was defined for calling the functions of external modules. During program start the functions used in the PDGL feature description are automatically entered into a function list via their PDGL keywords. This is realized by encapsulating the required funtcions into objects of a specific class. Objects of this class consist of a reference to the coded function, as provided by ACIS, and the corresponding PDGL keyword. The global definition of class objects enables them to add themselves to the list of PDGL functions during system start. In the case of the geometric modeller employed, ACIS, this is done for all functions of the procedural AP Interface. All functions carried in this list are treated as PDGL functions during interpretation. The feature modelling kernel is thus application-neutral in its implementation and is equipped with its application-specific functions at run-time, as presently implemented for functions of the geometrical modeller. The feature parameters required for instantiation are identified by the interpreter and specified by the user.

Corresponding requirements for the computer-internal representation of feature information and the architecture of the kernel required for feature processing result from the system-independent description of the features. The following components constitute the latter:

\subsubsection{PDGL Interpreter for the Generation of the Computer- Internal Model}

The interpreter for the processing of the textually described features is used for the generation of the computer-internal feature model during the modelling process. After selection of the features to be interpreted the parameters to be specified by the user are requested. The interpretation process subsequently undertakes the instantiation of the specific feature object in the computer-internal representation 
using the specified parameters. Functions used for the generation of the geometrical shape are initiated via the functional interface in the geometrical modeller described above.

\subsubsection{Product Structure for Semantically Oriented Processing of Feature Information}

For the computer-internal representation of the structure model a tree structure was developed that is designated Part Design Tree (PDT). In the PDT features are represented instead of geometrical primitives (cube, cone, etc.), in contrast to CSG models. In this way structure-related operations can take account of the semantics contained in the model. The Part Design Tree processes feature-nodes, transformation-nodes, combination-nodes and assembly-nodes.

Feature-nodes represent the features represented within the PDT. Transformation-nodes represent transformation features. Both types of nodes can only occur as end nodes within the tree. Assembly-nodes contain references to an arbitrary number of nodes which represent individual parts or sub-assemblies. A combination-node possesses two references to other nodes, as a rule. A name can be given to each node. Thus a sub-tree, whose root is this node, can be identified as an individual part.

\subsubsection{Feature Data Structure}

The use of generic features written in PDGL results in the generation of a computer-internal model. The structure underlying the model builds upon the object types defined in PDGL and makes possible the aggregation of several object types to one feature.

3.3.4. Bi-Directional Interface for the Processing of the Feature Geometry in the Geometrical Modeller

The bi-directional interface is used for the unambiguous separation of the feature and geometrical modellers. It makes possible controlled access on the part of each modeller to the data of the other modeller. This is implemented in each of the data structures by means of references to the corresponding data in the other data structure. For this purpose attributes are attached to each face of the geometric representation which points to its corresponding features. When features interact geometrically, functions of these attributes are called which update the feature geometry relation. Specifically, three cases have to be distinguished:

1 Deletion of a face, which causes the attribute function to delete the reference (and only the reference) from the corresponding feature object to geometry as well,

2 Split of a face, which causes the attribute function to inherit the reference of the corresponding feature object to the splitted faces and 
3 Merging of two faces, which is suppressed in the topological structure of the geometric modeller by initialisation parameters. This means that merged faces remain separated, although they have the same underlying mathematical description.

The references are updated automatically. The interface remains hidden to the user.

\subsubsection{Interfaces of the Feature Processing Module}

Access to the information contained in the computer-internal model proceeds via an object-related interface. Furthermore, functions required for the generation, linkage, copying and deletion of feature-nodes are made available via a procedural interface [EMJ 89]. Both the interface for information access and the procedural interface are semantically neutral. The evaluation of the semantics contained in the model is to be performed using the application module, Figure 3.

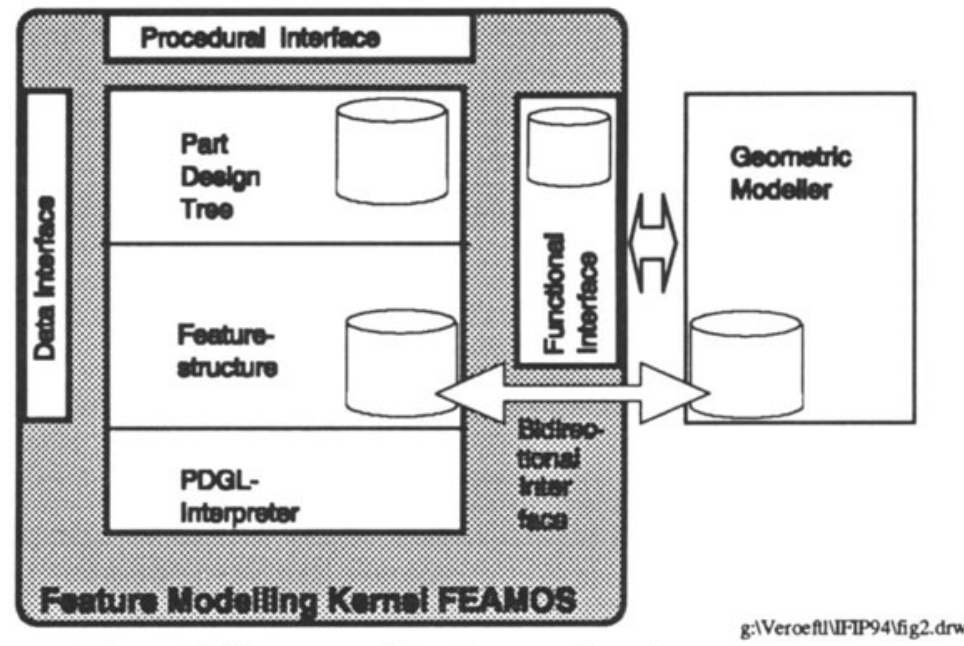

Figure 3. Structure of the Feature Kernel

\subsubsection{Rule based Feature Interpretation}

The efficient processing of feature information for the automation of design and planning processes requires the flexible representation of corresponding decision logics and their application-specific interpretation, e.g. for process sequence determination or tool selection [KRA 91], [HOU 91].

For the feature-based and user-oriented configuration and interpretation of process information a component was developed that supports the definition and processing of object structures, production rules and decision logics at run-time. During this process attributes of different types can be assigned to the objects, 
derived or generated. For the export and import of data an interface is provided by means of which external functions can be incorporated, e.g. for access to other databases, the feature modeller or for dialog. Through the definition of constraints ranges of validity for the objects and parameters are verified during access, Figure 4.

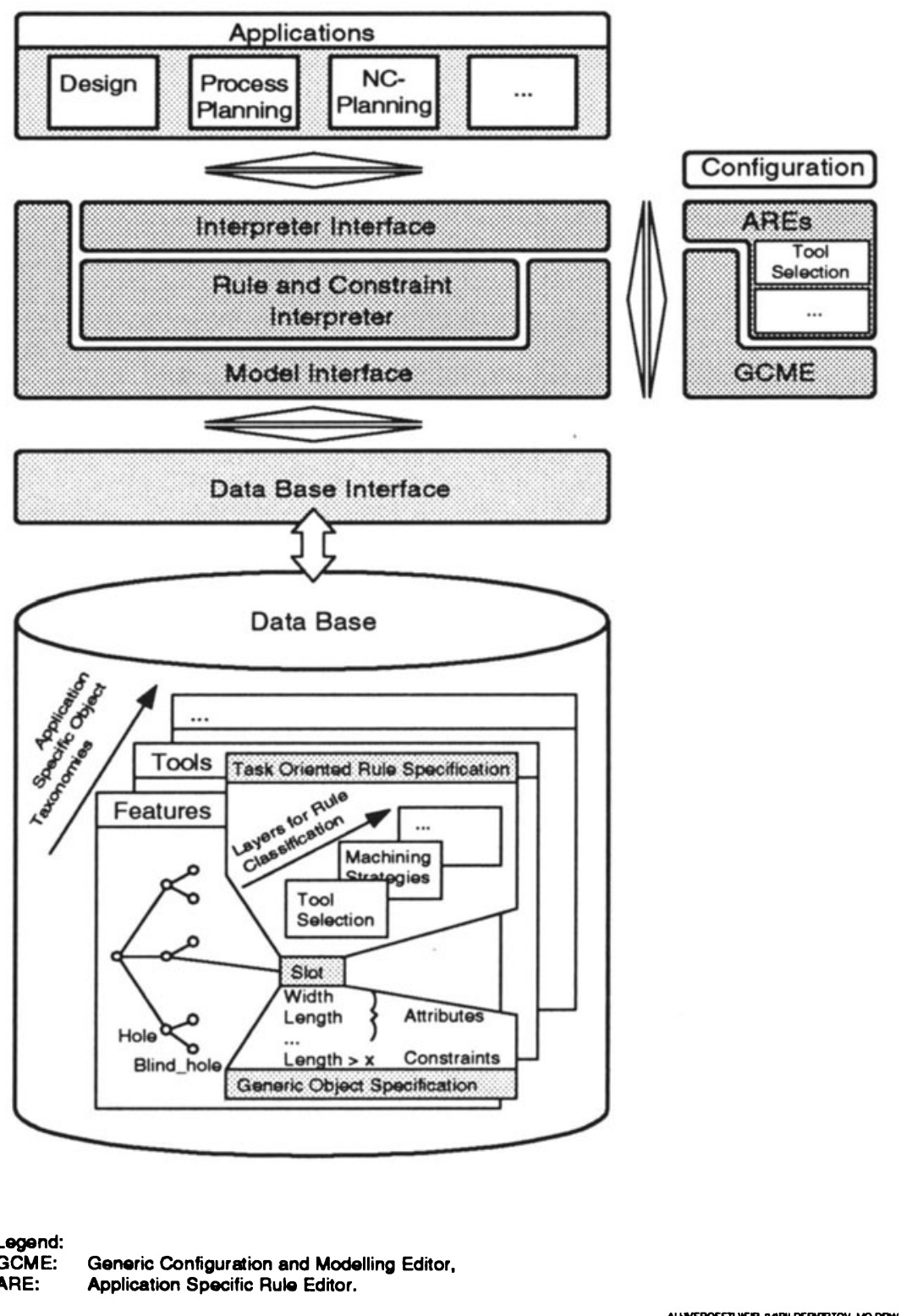

Figure 4. Architecture for Rule based Feature Interpretation. 
The representation of relationships between parameters of different object classes proceeds via rules, e.g. for the assignment of suitable processes to the manufacturing features. The context-specific differentiation of the rules for an object is supported using a layer concept. At each layer application specific rules, such as for tool selection, are attached to the corresponding specific feature objects. The individual layers are therefore to be activated application-specifically. In this way it is possible to distinguish the rules belonging to a feature in a taskoriented fashion.

Strategies for the evaluation of the logic represented in the decision trees, such as 'depth-first' or 'width-first' search, as well as rule processing, are implemented via the modelling of control rules.

The system consists of an interpreter and a database. The objects and rules are represented in the database in the form of a tree-like graph. The interpretation of the constraints and rules is performed by the interpreter. For access to the models two application-independent procedural interfaces are provided. Via the model interface conventional database access is performed, whereas the application interface is responsible for the interpretation of the data. Their functionality constitutes the basis for the realization of task-related, semantic interfaces. The implementation using client-server architecture supports the multi application, centralized delivery of generic as well as feature-related process information.

The configuration of the object structures and rules is performed in a graphicinteractive manner with an application-independent editor. For the efficient, taskoriented configuration of process information application-specific user interfaces are to be used. For this purpose program libraries are provided for the useroriented design of configuration interfaces.

The component represents a kernel consisting of different libraries and to be configured task-specifically for the development of application systems. It is to be regarded as a black box and is used at the present time for feature management and interpretation, delivery of process information as well as transformation of control-independent NC information into control-specific NC programs.

\subsection{SUPPORT OF CAE - FUNCTIONALITY THROUGH FEATURES}

\subsubsection{Elementary Features as Integration Elements}

The semantic integration of tasks in the product development process proceeds on the basis of the computer-internal representation of the feature model as well as of manufacturing-related elementary features, such as slots, pockets or holes. Elementary features are company- and process-specific objects about which, however, the certain determination has been made of their manufacturability with available tools and machines by means of one or several processes. Through their use a product-design in tune with manufacturing needs is in large measure to be secured. They form the application-specific extension of the Form Feature Model 
according to ISO 10303 [ISO 92b], [SHM 91].

The application-specific feature definition for design or planning tasks proceeds either via configuration of the elementary features or via derivation of task-oriented feature classes. In both cases the completition of application-specific information is performed.

The complex design objects used for functional design and the increase of efficiency of the design process are composed of elementary features. In the generation of feature instances during the design process the information concerning elementary elements is retained in the feature model by means of which the basis for integration is created. Management of design objects is performed by the PDT.

In contrast to the design process both, the parameterized as well as the explicit description of the machining volumes, of the machining feature and their machining sequence, are necessary for process-planning. For this reason the elementary features to be transferred must be identified and their geometrical relationships among each other determined, Figure 5. For this purpose both the feature and the geometrical models are analyzed. The identification of the elementary features relevant to manufacturing is performed by making a check of all feature instances against the reference structure for the management of elementary features, which is also used for the selection, management and configuration of application-specific features. By means of geometrical analysis the spatial relationships between features are determined. The result is represented in the "Part Manufacturing Tree (PMT)" which is generated during feature mapping between design and process planning. The PMT is a graph-oriented precedence structure in whose nodes the references to the elementary feature instances in the feature model relevant to machining are represented [ANC 90a], [ANC 90b]. The spatial relationships of the features among one another are represented by links between nodes. Links are relations that more precisely specify the topological dependencies [LAA 93b].

Taking account of the blank part shape a process-independent generation of the machining volumes for the machining features is performed. Machining elements that do not come into being until the creation of differential geometry are not derived from the description of the finished part but rather interactively identified and integrated into the PMT.

The subsequent process-oriented interpretation of the machining features leads to the feature-specific determination of alternative process sequences, composed of process elements. To each process element belong operation elements with corresponding cutting volumes. They constitute the basis for the generation of NC paths. The sum of the cutting volumes of an operation sequence yields the machining volume of a process element.

\subsubsection{Definition of New Features}

To achieve user independence from the form of textual description of the features the definition of new features can be undertaken in a graphic-interactive fashion. 
The user designs the feature to be defined using the feature modelling functionalities at his disposal. All previously defined features are available to him during this process. The definition as feature is then performed by selecting the corresponding PDT-tree. Subsequently the function for the definition of new features is initiated. The system ascertains the required parameters for the instantiation of the new feature. The user has the option here of giving the parameters new names since they are part of the feature semantics, or assigning them fixed values. Finally, the type of the newly defined feature must be specified, whereupon the appropriate PDGL description is generated. The generated feature remains available during the current program run for the continuing design process. Beyond the targeted definition of a new feature it is possible at a later point in time to derive a feature definition from a partial tree of the model.

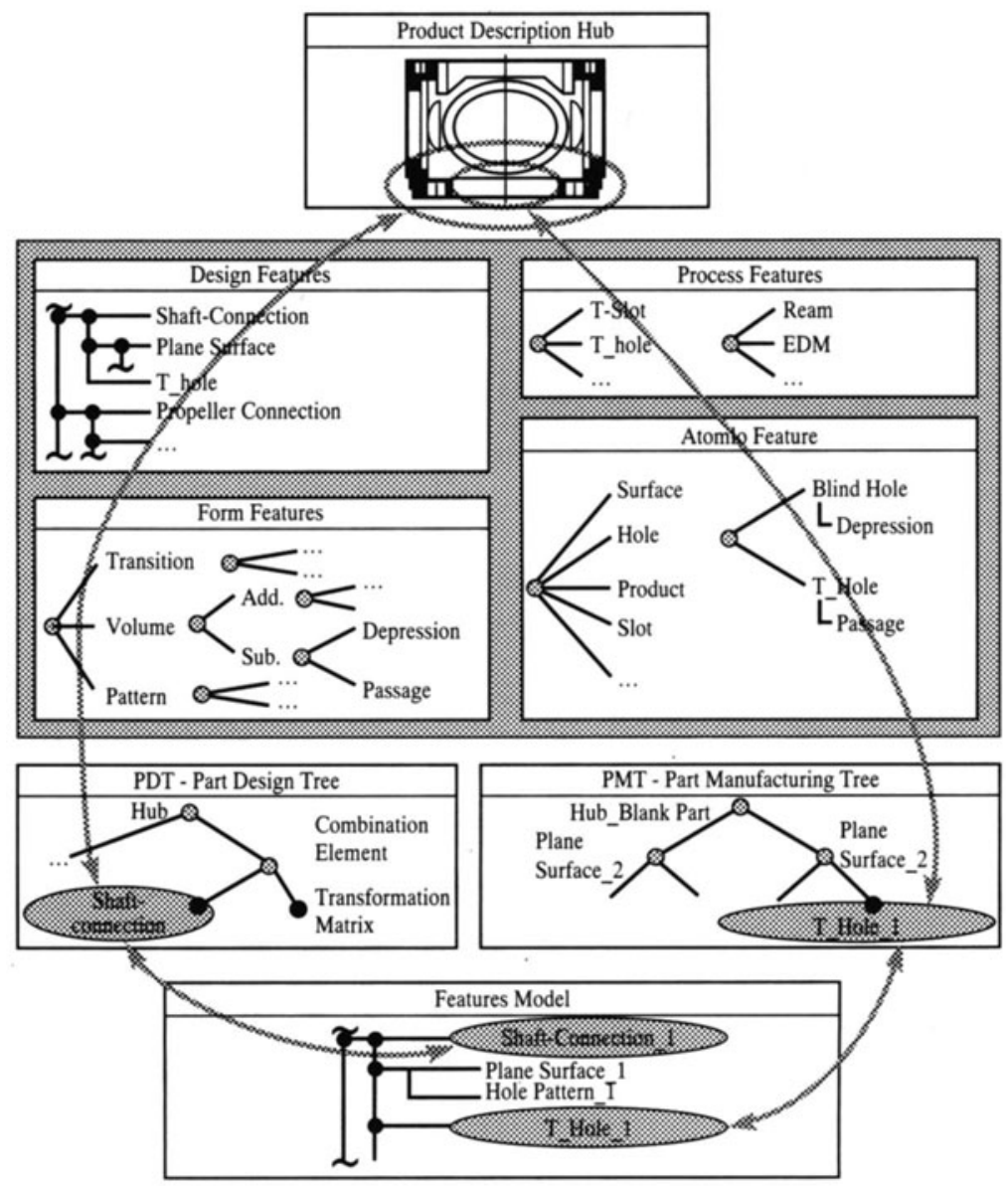

Figure 5. Integration on the Basis of Elementary Features 


\subsubsection{Extraction of Feature Parameters with PDGL}

For the application-specific definition of features that cannot be derived from design features or that relate to an intermediate work-piece state and not to the finished part the provision of methods for feature recognition is necessary, e.g. for quality or fixture planning [DVW 91]. For the identification of features, functions for geometrical analysis are required. These can either be rigidly implemented or task-specifically configured with the aid of an interpretable description language. Hard-coded methods for the identification of features can only be complemented and modified by means of programming changes [BHR 89], [JOC 88]. A more flexible alternative is, however, offered by the use of PDGL. Since the essential elements of a programming language are made available by PDGL, an extension is necessary only with respect to the required basic functions for geometrical analysis. PDGL is extended by these basic functions using the functional interface described earlier.

The use of PDGL makes possible the automated recognition of specific features through the analysis of the complete part or the graphic-interactive identification of the features and the automated determination of their descriptive parameters [RVE 89].

Graphic-interactive feature definition can be used efficiently for the support of dedicated applications since the complexity of both the descriptive and analytic overhead is significantly reduced.

The description of the analysis sequence is performed with PDGL by extending it to include the corresponding methods for the derivation of feature parameters, Figure 6.

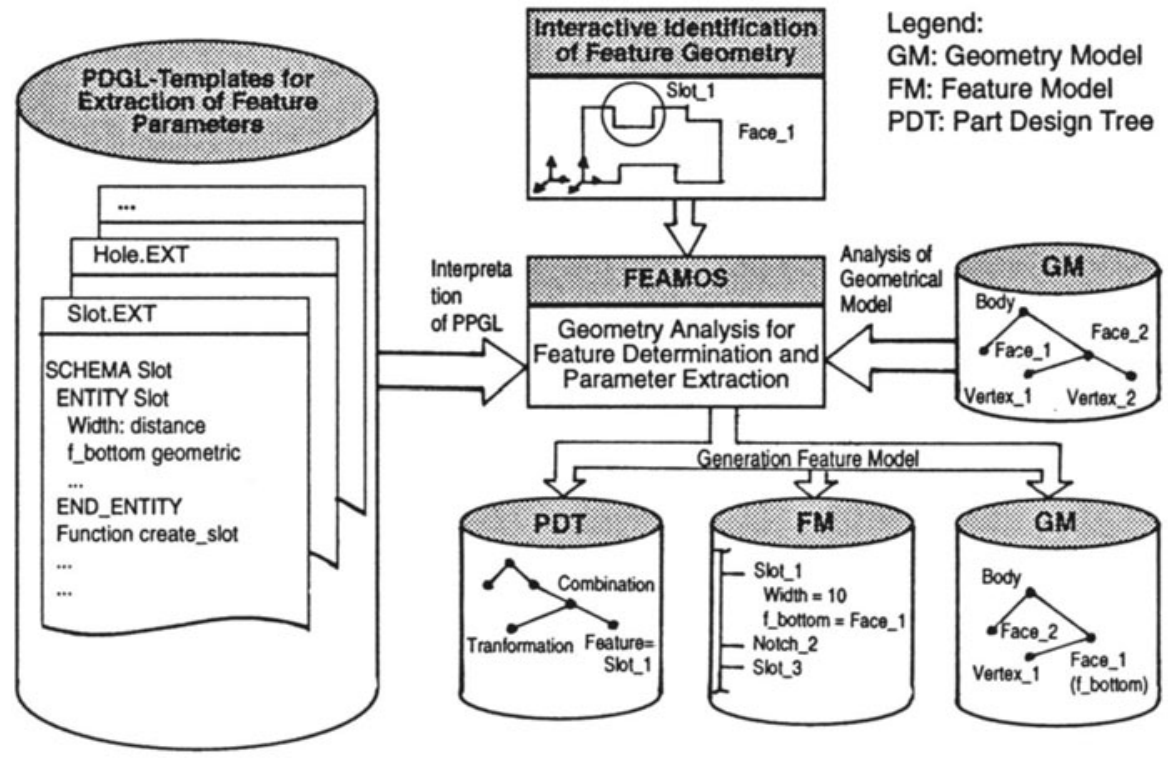

Figure 6. Extraction of Feature Parameters. 
For this purpose the surfaces describing the feature, their parameters, as well as position and orientation of the feature with respect to the workpiece origin are determined from the computer-internal representation of the geometrical model. To support subsequent planning tasks semantic elements relevant to planning, e.g. the characterization of side and bottom surfaces are added to the surfaces via attributes. The agreements defined in the Form Feature Model of ISO 10303 (Product Data Representation and Exchange) are used as the basis [ISO 92b], [WIP 88]. At the same time the bi-directional interrelation between feature and geometrical model is established via the attributes and the positions of the local reference systems are defined. After the determination of all parameters the feature is represented in the feature model [HOU 91].

The essential methods for parameter extraction are categorized according to their purpose as functions for the determination of:

1 Geometrical attributes (surfaces and contour shapes),

2 Topology (adjacent surfaces and surface transitions)

3 Parameters (distances, angles) or

4 Vectors (normal, direction)

The methods are superimposed upon basic functions that access the computerinternal representation via the model interface of the geometrical modeller and determine geometrical information such as e.g. edges of a surface or all surfaces parallel to a surface [BHA 88], [LAA 93a].

The interactive definition of features is presently being used in planning preparation for the transfer of geometric differential volumes into machining features and in fixture planning for the definition of clamping and bearing faces in the current intermediate work-piece state, not on the finished part, for the support of the selection and configuration of clamping devices.

\subsubsection{Feature Mapping}

The goal of feature mapping is the process independent ascertainment of all elementary elements implicitly established by design as well as the determination of their initial states taking account of the blank part shape [SHA 88a], [SHA 88b] [LMM 90].

The transformation of design into machining features proceeds by scanning the feature model. All identified elementary features as well as their geometrical interrelations are transferred into the PMT. The nodes of the PMT refer to the instances of the elementary features in the feature model. By means of the references the distinction can be made between predecessor and successor elements as well as geometry-dependent and independent alternatives.

The ascertainment of the relationships of the features among each other proceeds via analysis of the geometry model representing the form feature semantics, such as the characterization of side or bottom face [ISO 92b], [SHM 91]. Features possessing several predecessors through geometrical relationships are 
unambiguously assigned by the user. Features to which no geometrical relationships among each other are available are represented in the PMT as alternatives. The automatically generated PMT structure can subsequently be graphically-interactively completed or modified.

In the following step the cutting volumes of all elementary features are determined taking account of the blank part geometry. For this purpose the PMT is scanned with a backwards orientation. For each feature the corresponding cutting volume is generated, in which at for each instance the intersection with the blank part is taken and represented in the feature model.

Subsequently, all cutting volumes are united with the finished part. If a difference remains between blank and finished part the decomposition of the corresponding machining volume proceeds graphically-interactively. The machining features that are created are, to the extent that their shapes match the corresponding elementary features, complemented with a parameterized description. In addition these elements are graphically-interactively identified as features and their specific parameters automatically extracted.

\subsubsection{Process Feature Description with PDGL}

Process feature description is used in the determination of process-specific cutting volumes dependent on material, the surface quality demanded and the machining offsets. The goal is the generation of operation elements whose cutting volume is to be machined with completely one tool. Above and beyond this, information relevant to planning can be directly assigned to every process feature, e.g. with respect to approach or machining directions [MOP 89].

Several process features are therefore assigned to each elementary feature, such as mill_pocket or erode_pocket. Using rule-based interpretation of the parameters of a feature instance all suitable processes are determined. The suitable process sequences are generated backwards-oriented proceeding from final machining process and are based on the process independent machining elements generated during feature mapping, Figure 7.

For machining processes, involving material removal, the machining offsets are determined dependent upon information about surface roughness. The determination of the current offsets of the process independent machining features is subsequently performed. For each bottom and side face the maximum distance to the next positioned face is determined.

In cases involving features with offsets, such as e.g. pre-manufactured or cast components, the current offset of each face is compared with the required offset. If the current offset lies within a defined tolerance range the required offset is replaced with the current offset. This leads to the result that no predecessor feature and thus no predecessor process is generated. By means of such rules a differentiated manipulation of the machining features contingent upon material classes is supported.

If, however, a predecessor process is required, the parameters for the remaining volumes are computed for the resulting feature type hased on the 
maximum offsets. The generation of the corresponding feature instance constitutes the prerequisite for the ascertainment of the predecessor processes as well as for the computation of the cutting volume of the current feature by subtracting the remaining volume from the elementary feature.

The determination of the machining processes is subsequently performed for the newly created feature through interpretation of its parameters which are obtined from the corresponding PDGL-description.

In this way a structure with alternative process sequences is created for every elementary feature. The alternative machining processes are represented in the feature model corresponding to the cutting volumes [ALT 91].

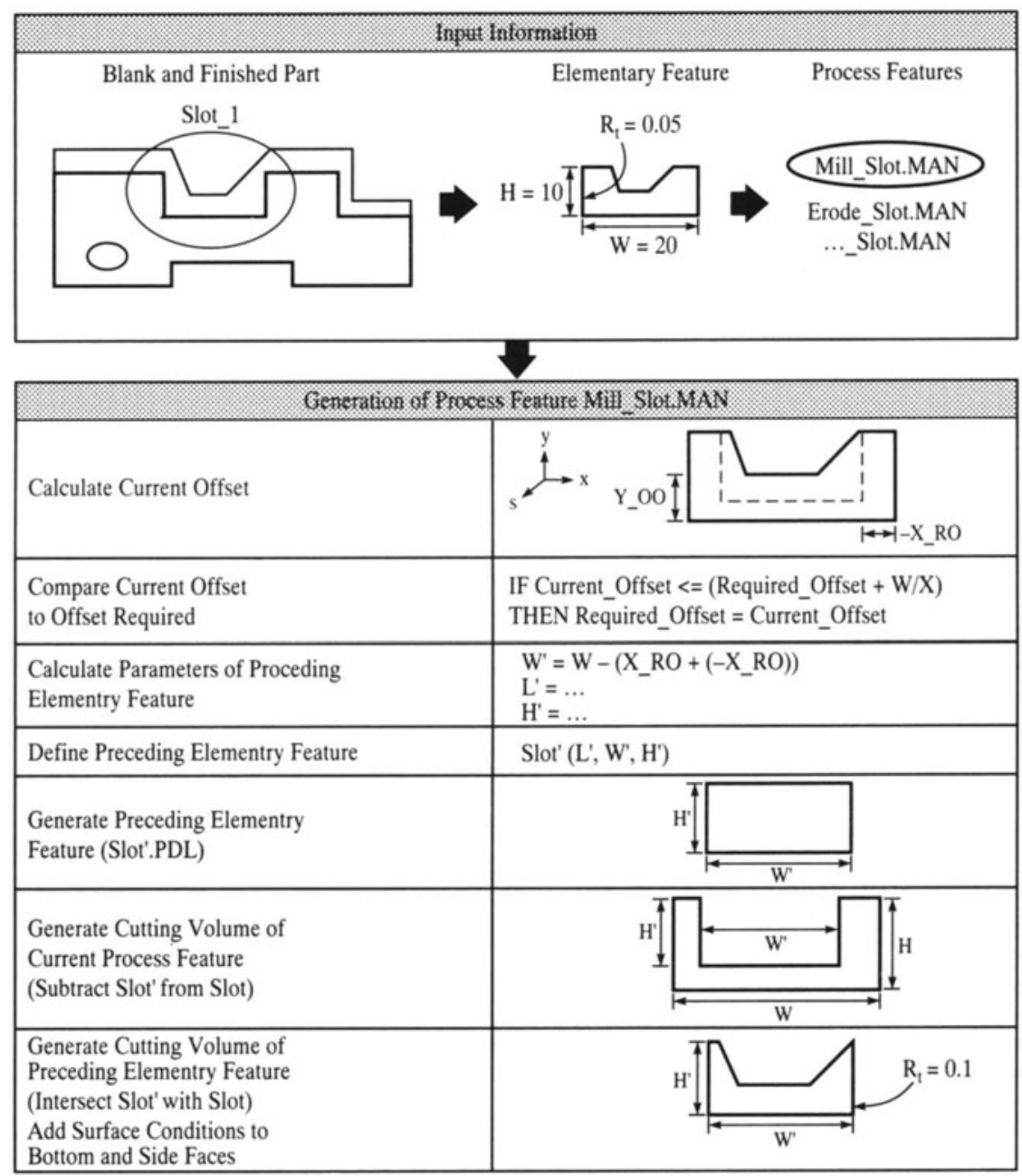

Legend: $-\mathrm{X} \_\mathrm{RO}:$ Required Offset in Direction $(-\mathrm{X})$

$-Y_{-}$OO : Current Offset in Direction $(+Y)$

Figure 7. Generation of Process Features. 


\subsection{FEATURE LIBRARY MANAGEMENT}

A consistent management of generic and specific features is required for the use of features in an environment involving many applications. A suitable functionality is therefore be offered that supports information delivery via an application interface. For the management of features a user interface is provided by means of which the user can define, configure and - in application-specific taxonomies for design, computation and planning support - integrate new features, Figure 8.

The basis for information delivery is formed by reference structures. In specific, they contain:

1 the location of storage of the generic feature,

2 the designation of explicit and implicit parameters as well as their data types,

3 the basic elements of application-specifically configured features,

4 pre-defined instances of the parameters of specific features, and

5 information about their application

For the representation of the reference structures a database was applied, and a client-server concept was used for implementation. In this way it is made possible for the different modules to access information contained in the database independently of one another.

For the central management and maintenance of the feature data basis a configuration interface was implemented that supports the graphic-interactive definition and manipulation of the information objects.

When extending the data basis by adding new features, the determination of the feature parameters as well as the elementary features used from the corresponding PDGL description is performed automatically after input of the location of storage of the new feature. By means of these mechanisms the consistent representation of the feature information is insured.

\subsection{OUTLOOK}

In the present contribution an implementation of a feature processing kernel has been introduced. The semantic integration of the application components is thus supported by the continuous use of features. The feature processing kernel serves as a central functionality for generating and modelling products in terms of features. Within several projects different applications have been realized on top of the feature kernel. These modules are listed in figure 1, a more detailled description can be found in [KCR 94].

The feature modelling kernel has reached a status which is sufficient for its commercialisation. This is currently in progress within the framework of an industrial cooperative effort. 

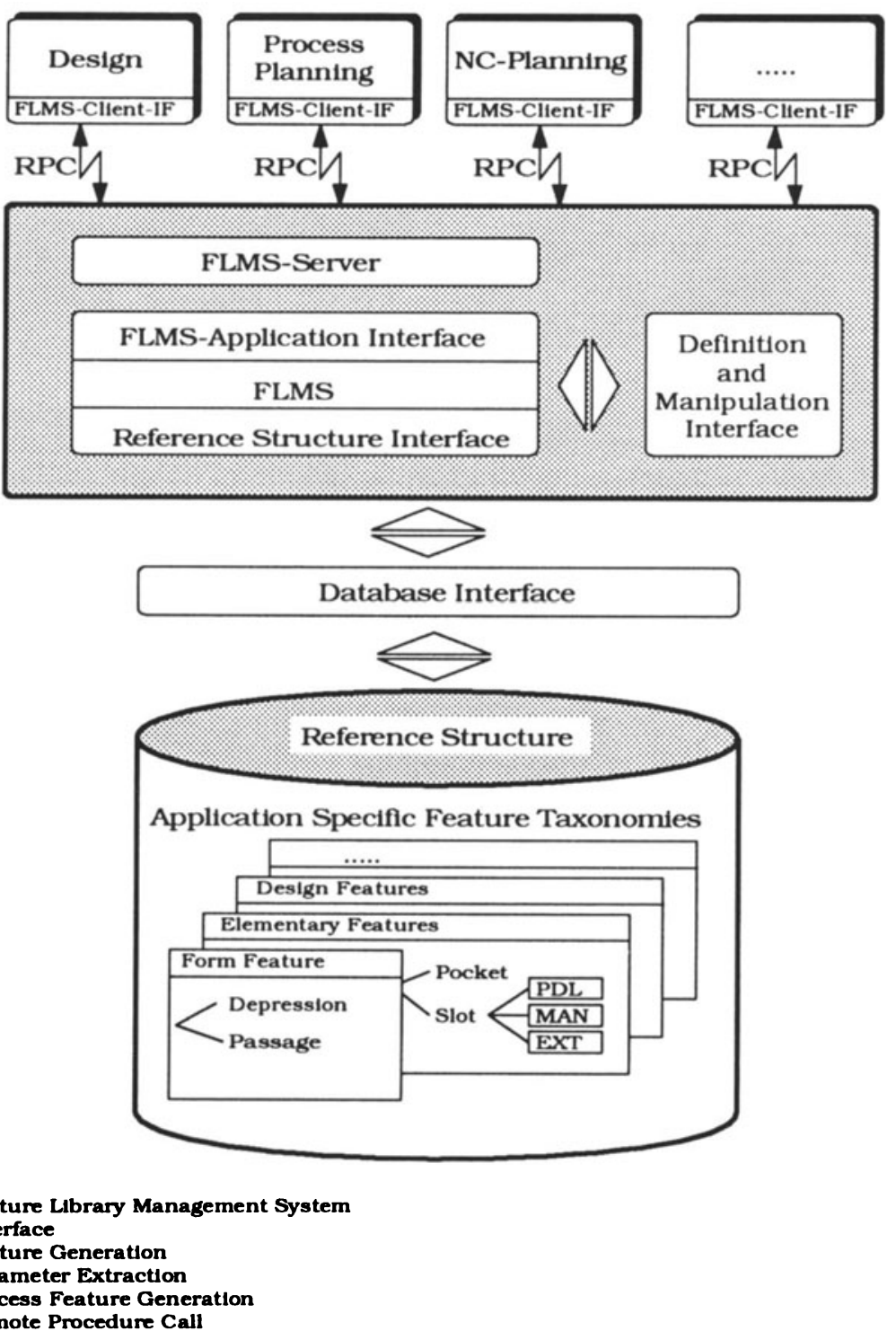

Legend:

FLMS Feature Library Management System

IF Interface

PDL Feature Generation

EXT Parameter Extraction

MAN Process Feature Generation

RPC Remote Procedure Call

Figure 8. Architecture of the Feature Library Management System

At present the feature approach is applied to the computer-aided application of the Quality-Function-Deployment (QFD) Method. Here customer wishes are translated into technical requirements, with the support of functional modelling using features. The generation of the product shape is performed by ascertaining a suitable solution in correspondence with the functional requirements from among a number of alternative solutions and transforming it into design features. The results of the QFD method subsequently control the quality assurance process accompanying product development, making use of product and process FMEA on 
the basis of quality features [KUW 93].

For the industrial deployment of feature-based systems the development of neutral models for the exchange and representation of product-defining data is necessary. Within the framework of ISO 10303 "Product Data Representation and Exchange" [ISO 92b] the model for the description of form features is presently to be found in the stage of the standardization process. Current activities consist in the development of an application protocol for the representation of mechanical components based on the form feature model. In the area of parametric modelling there exists, however, a great need for future standardization.

\subsection{REFERENCES}

[ALT 91] AltmanN, Ch.: Dynamische Prozeßgestaltung für flexible Fertigungssysteme durch integrierte Arbeitsvorbereitung, München, Wien: Hanser, 1991.

[ANC 90a] Anderson, D.C.; Chang, T.C. : Automated Process Planning Using ObjectOriented Feature Based Design, Advanced Geometric Modelling for Engineering Applications, edited by F.-L. Krause and H. Jansen, Elsevier, IFIP/GI, 1990.

[ANC 90b] ANDERSON, D.C.; Chang, T.C.; Kak, A.; Mitchell, O.R.: A Quick Turnaround Cell, CAM-I Report No. P-90-PM-02, 1990.

[BHA 88] BhatnaGAR, A.S.: Implementation of Feature Mapping and Reasoning Shell with Application to Group Technology Coding, MSc Thesis, Anzona State University, August 1988.

[BHR 89] Billo, R.E.; Henderson, M.; Rucker, R.: Applying Conceptual Graph Inferencing to Feature-Based Engineering Analysis, Computers in Industry, Vol. 13, 1989, pp. 195-214.

[BJØ 92] BJøRKE, O. (ed.); Myklebust, O. (ed.): IMPPACT - Integrated Modelling of Products and Processes using Advanced Computer Technologies, Tapir Publishers, Trondheim, 1992.

[BRA 88] BRAID, I.C.: Improving Product Models and Kernel Modellers, Proceedings of the 2nd Toyota Conference, Aichi, Japan, Oct. 2.-5., 1988, pp. 275 - 302.

[COF 92] CocQueberT, E.; Féru, F.; Chouch, H.; Denuex, D.; Soenen, R.: State of the Art and Evolution in Feature-Based Modelling, Revue internationale de CFAO et d'Tnfographie, Volume 7-nº 2/1992, pp. 169 à 200.

[DVW 91] Dong, X.; DeVries, W.R.; Wozny, M.J.: Feature-Based Reasoning in Fixture Design, Annals of the CIRP, Vol. 40, No. 1, 1991, pp. 111-14.

[EMJ 89] VAN EMMERIK, M.J.G.M.; Jansen, F.W.: User Interface for Feature Modelling, Computer Applications in Production and Engineering, edited by F. Kimura and A. Rolstadas, Elsevier, IFIP, 1989.

[HOU 91] van Houten, F.J.A.M.: PART: A Computer Aided Process Planning System, PhD Thesis, University of Twente, Enschede, 1991.

[HOU 93] van HouTEN, F.J.A.M. van; Salomons, O.W.; Kals, H.J.J.: Review of Research in Feature-Based Design, Journal of Manufacturing Systems, Vol. 12, No. 2, pp. 113 132, 1993.

[ISO 92a] N.N.: ISO WD 10303-48 Product Data Representation and Exchange - Part 48 Form Features, ISO TC 184 / SC4 / N102, Januar 1992.

[ISO 92b] N.N.: ISO DIS 10303-11 Product Data Representation and Exchange - Part 11 The EXPRESs Language Reference Manual, ISO TC 184/SC4/N151, August 1992. 
[JOC 88] Josh, S.; Chang, T.-C.: Graph-Based Heuristics for Recognition of Machined Features from a 3-D Solid Model, Computer-Aided Design, Vol. 20, No. 2, 1988, pp. 58-64.

[KCR 94] KRAUSE, F.-L.; Ciesla, M.; Rieger, E.; Stephan, M.; Ulbrich, A.: Features als semantische Objekte integrierter Prozeßketten, Tò be presented at the GI - WG 5.2 Conference: $\mathrm{CAD}^{\prime} 94$ - Produktdatenmodellierung und Prozeßmodellierung als Grundlage neuer CAD-Systeme, March, 17-18th 1994.

[KKR 91] KRAUSE, F.-L.; Kramer, S.; Rieger, E.: PDGL - A Language for Efficient Feature Based Product Gestaltung, CIRP Annals, Vol. 40/1, PP. 135-138, 1991.

[KRA 91] KRAUSE, F.-L.; Altmann, C.; Integration of CAPP and Scheduling for FMS, in Doumeingts, G., Browne, J., and Tomljanovich, M. (eds.), Computer Applications in Production and Engineering: Integration Aspects (Proc. CAPE 91), North-Holland, Amsterdam, 1991, pp. 535-541.

[KRC94] KRAUSE, F.-L.; Ciesla, M.: Technologische Planung von Meßprozessen für KoordinatenmeBmaschinen, ZwF 89 (1994) 3, pp. 133-135.

[KUV 90]Krause, F.-L.; Ulbrich, A.; Vosgerau, F. H.; Feature based Approach for the Integration of Design and Process Planning Systems. 23rd International Symposium on Automotive Technology \& Automation, Proceedings 1990.

[KUW 93] KRAUSE, F.-L.; Ulbrich, A.; Woll, R.: Methods for Quality-Driven Product Development, Annals of CIRP Vol. 41/1, pp. 151-154, 1993.

[LAA 93a] LAAKKo, T.: Incremental Feature Modelling: Methodology for Integrating Features and Solid Models, PhD Thesis, Helsinki University of Technology, Finland, October 1993.

[LAA 93b] LAAKKo, T.; Mantylä, M.: Feature Modelling by Incremental Feature Recognition, Comp. Aided Design, Vol. 25 (8), pp. 479-492, August 1993.

[LMM 90] LAAKKo, T.; Mäntylä, M.; Mäntylä, R.; Nieminen, J.; Sulonen, R.; Tuomi, J.: Feature Models for Design and Manufacturing, 23rd Hawaii International Conference on System Sciences, IEEE, Vol. II, edited by B.D. Shriver, 1990, pp. 445-54.

[MOP 89] MÄntYlÄ, M.; Opas, J.; Puhakka, J.: Generative Process Planning of Prismatic Parts by Feature Relevation, Advances of Design Automation 1989, presented at the ASME Design Technical Conference - 15th Design Automation Conference, Montreal Quebec, Canada, 1989, pp. 49-60.

[PRA 90] PRATT, M.J.: A Hybrid Feature Based Modelling System, Advanced Geometric Modelling for Engineering Applications, edited by F.-L. Krause and H. Jansen, Elsevier Science Publishers B.V. (North Holland), 1990, pp 189-201.

[REV 89] ReQUICHA, A.A.G.; Vandenbrande, J.H.: Form Features for Mechanical Design and Manufacturing, Proceedings ASME International Computers in Engineering Conference (CIE), Anaheim CA. 1989, pp. 47-52.

[SHA 88a] SHAH, J.J.: Feature Transformations Between Application-Specific Feature Spaces, Computer Aided Engineering Journal, December 1988, pp. 247-55.

[SHA 88b] SHAH, J.J.; Bhatnagar, A.; Hsiao, D.: Feature Mapping and Application Shell: Computers in Engineering Conference (CIE), San Francisco CA, 1988, pp. 489-96.

[SHA 91] SHAH, J.J.: Conceptual Development of Form Features and Feature Modellers, Research in Engineering Design, Vol. 2, 1991, pp. 93-108.

[SHA 91] SHAH, J.J.: Assessment of Features Technology; Computer Aided Design, Vol 23, Jun 1991.

[SHM 91] SHAH, J.J.; Methew, A.: Experimental Investigation of the STEP-Form Feature Information Model, CAD, Vol. 23, No. 4. 1991. 
[SRS 90] SHAH, J.J.; Rogers, M.T.; Sreeyalsan, P.C.; Hsiao, D.; Mathew, A.; Bhatnagar, A.; Liou, B.; Miller, D.W.: The ASU Features Testbed: An Overview, ASME Computers in Engineering Conference (CIE), 1990, pp. 233-41.

[WIP 88] WILSON, P.R.; Pratt, M.J.: A Taxonomy of Form Features for Solid Modelling Geometric Modelling for CAD Applications, edited by M.J. Wozny and H.W. McLaughlin, Elsevier Science Publishers B.V. (North Holland) IFIP, 1988, pp. 125-35. 